\title{
A 5-6 GHz 1-V CMOS Direct-Conversion Receiver With an Integrated Quadrature Coupler
}

\author{
Hsiao-Chin Chen, Tao Wang, and Shey-Shi Lu
}

\begin{abstract}
This paper describes a novel monolithic low voltage (1-V) CMOS RF front-end architecture with an integrated quadrature coupler (QC) and two subharmonic mixers for direct-down conversion. The $L C$-folded-cascode technique is adopted to achieve low-voltage operation while the subharmonic mixers in conjunction with the QC are used to eliminate LO self-mixing. In addition, the inherent bandpass characteristic of the $L C$ tanks helps suppression of $L O$ leakage at RF port. The circuit was fabricated in a standard 0.18- $\mu \mathrm{m}$ CMOS process for 5-6 GHz applications. At 5.4 GHz, the RF front-end exhibits a voltage gain of $26.2 \mathrm{~dB}$ and a noise figure of $5.2 \mathrm{~dB}$ while dissipating $45.5 \mathrm{~mW}$ from a $1.0-\mathrm{V}$ supply. The achieved input-referred DC-offset due to LO self-mixing is below $-110.7 \mathrm{dBm}$.
\end{abstract}

Index Terms-Direct-down conversion, folded cascode, front-end, homodyne receivers, LO self-mixing, low voltage operation, low-noise amplifiers, mixers, RFIC.

\section{INTRODUCTION}

$\mathbf{I}$ N RECENT years, the direct-conversion topology has become the choice for receiver architecture in many wireless transceivers due to its low complexity and high potential for system-on-a-chip (SOC) applications. Nevertheless, there are several issues to be solved in this popular architecture. The DC-offset originating from local oscillator (LO) self-mixing phenomenon in direct-conversion mixers may be the most difficult one among all these issues. For conventional direct-conversion mixers, the required $\mathrm{LO}$ frequency is equal to the input radio frequency (RF). If the isolation between the LO port and RF port is insufficient, part of the LO signal will enter the RF port via any possible paths. When this so-called LO-to-RF leakage is multiplied with the original LO in the mixer, an output component at zero-frequency will appear and behave like DC-offsets. For direct-conversion receivers, the DC-offset at mixers output should be as low as possible because it not only degrades the signal-to-noise ratio (SNR) but also prohibits the signal amplification in the following analog baseband circuits (ABBs).

To eliminate LO self-mixing DC-offset, preventing the use of a LO frequency from being equal to RF may be the most straightforward solution and this concept brings forth

Manuscript received August 8, 2006; revised March 21, 2007. This work was supported by the National Science Council under Contract NSC 94-2215-E002-032 and the Ministry of Economic Affairs of the R.O.C. under Contract 94-EC-17-A-05-S1-0017.

The authors are with National Taiwan University, Taipei 10617, Taiwan, R.O.C. (e-mail: sslu@cc.ee.ntu.edu.tw).

Digital Object Identifier 10.1109/JSSC.2007.903041 the subharmonic mixers (SHMs) which utilize LO signal at half (or third, fourth, fifth, etc.) of the RF input frequency. Historically, harmonic mixing using passive devices was proposed for applications at the higher millimeter-wave frequencies where reliable stable LO sources are either unavailable or prohibitively expensive [1], [2]. Recently, various active SHMs were proposed as candidates for direct-conversion mixers [3]-[8]. However, they are hardly adequate for low-voltage operation, which may become an obstacle to system integration in advanced digital IC processes. Furthermore, the LO-to-RF isolation has not been particularly handled in most of these previous works. In fact, the isolation issue is very important as it relates to the leakage of $\mathrm{LO}$ and $2 \times \mathrm{LO}$ at RF port of SHMs. For direct conversion receivers utilizing SHMs, large LO leakage still leads to DC-offset due to circuit imperfection and the $2 \times \mathrm{LO}$ leakage lying in the receive band could be picked up by antennas of nearby receivers or the original receiver from which it reradiates [5], [7].

Theoretically, excellent LO-to-RF isolation is an inherent property in a doubly balanced mixer and LO rejection (LOR) [3], [5] can be provided by a SHM. Therefore, it is conceivable that the LO leakage and the DC-offset due to the LO self-mixing can be diminished down to the noise level in any doubly balanced SHM. Unfortunately, such advantage is seldom attained in most practical situations since any mismatches between devices or differential signal paths could easily destroy the symmetry of the circuit. Thus, the ideal condition would not exist and the amount of the LO leakage or DC-offset depends on how large the asymmetry is. It is well known that it is difficult, if not impossible, to keep all the devices perfectly matched in standard IC processes. Therefore, in this work a technique offering extra LO-to-RF isolation and LOR is used to mitigate the LO self-mixing DC-offset and the $2 \times \mathrm{LO}$ re-radiation problem in SHMs rather than overly demanding the symmetry of circuits.

This work presents a novel 1.0-V 4.9-5.9-GHz monolithic CMOS receiver front-end exhibiting high LO self-mixing rejection. The rest of this paper is organized as follows. In Section II, the receiver architecture will be briefly described. In Section III, a new low-voltage SHM and some related concepts will be elaborated. The design of a low-noise amplifier (LNA), the on-chip quadrature coupler (QC) and transformers will also be mentioned. The experiment results will be reported in Section IV followed by a conclusion in Section V.

\section{RECEIVER ARCHITECTURE}

Fig. 1 shows the block diagram of the proposed direct conversion receiver consisting of an LNA, an on-chip QC, on-chip 


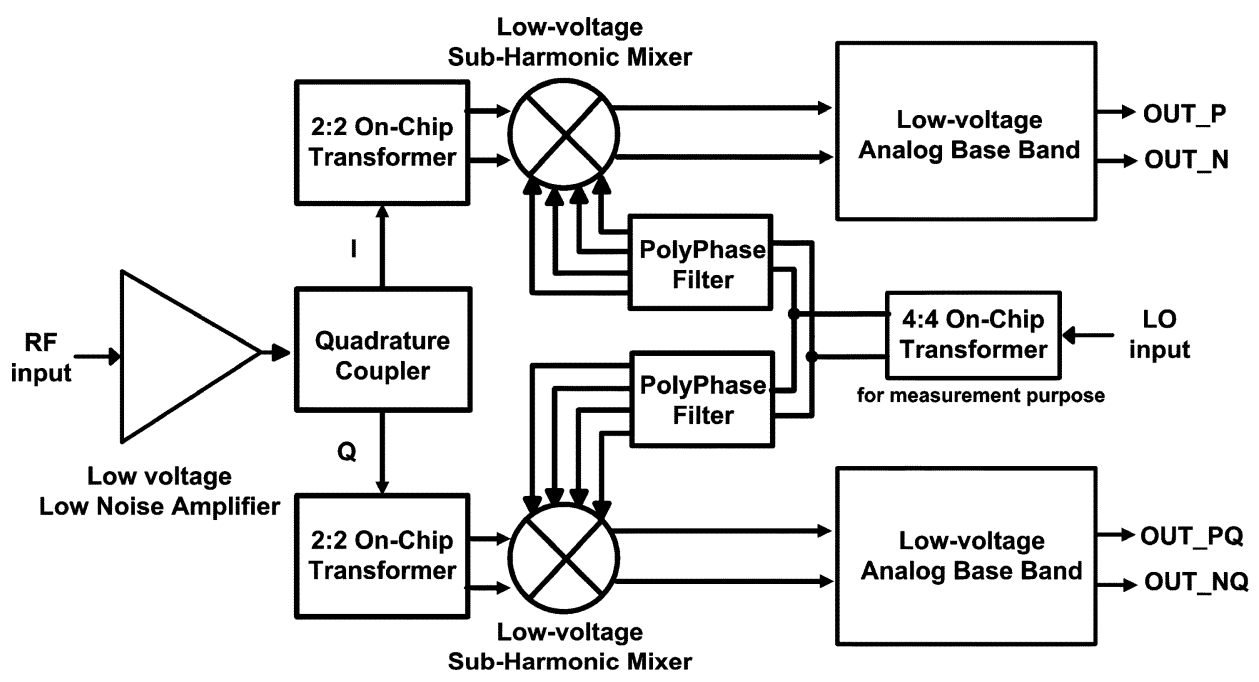

Fig. 1. Block diagram of the direct conversion receiver.

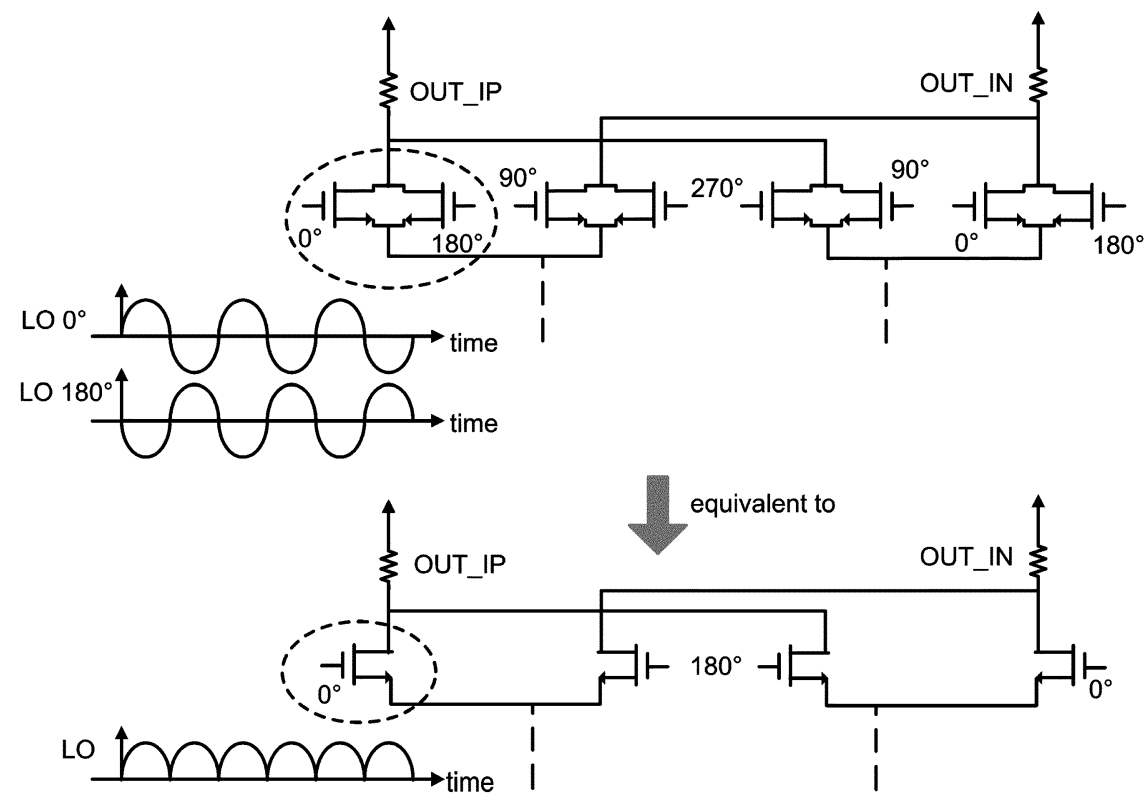

Fig. 2. Transformation of a subharmonic mixer from a conventional Gilbert mixer.

transformers, poly-phase filters (PPFs), SHMs and ABBs. First note that simple $90^{\circ}$ phase splitting of the subharmonic LO signal can only achieve differential output in SHMs and hence we use the on-chip integrated QC to perform RF path quadrature generation so that the complex octet-phase generation circuits [8] become unnecessary. Following the single-ended lowvoltage folded-cascode LNA, the QC splits the RF input signal into two signals in quadrature which are then converted into differential signals by the transformers in front of the low-voltage SHMs. Entering the doubly balanced SHMs, these differential signals are multiplied with the subharmonically pumped LO signal to accomplish signal down conversion. The in-band component of down-converted signal can be further amplified in the ABBs while the out-of-band interferer would be effectively suppressed. This work is focused on the RF front-end circuits ahead of ABBs and all the circuits are designed for low voltage operation.

\section{Circuit Design}

\section{A. Low-Voltage Subharmonic Mixer}

The principle of a SHM is briefly explained with Fig. 2 [9]. A conventional Gilbert mixer is transformed into a SHM by altering the configuration of the switching quads in a procedure described as follows. Each single-transistor switch in the Gilbert mixer is replaced by a dual-transistor switch, in which the two transistors are connected in parallel. From Fig. 2, it is clear that the case of applying two mutually out-of-phase signals to the dual-transistor switch is equivalent to that of turning on a single-transistor switch with doubled-frequency of the applied signals. Thus, direct down-conversion in the SHM can be achieved with LO signals operating at half RF frequency. Note that the proposed method can be further extended. That is, if an $\mathrm{n}$-transistor switch is used, where the $\mathrm{n}$ transistors are connected in parallel, direct down-conversion in the SHM can be achieved 


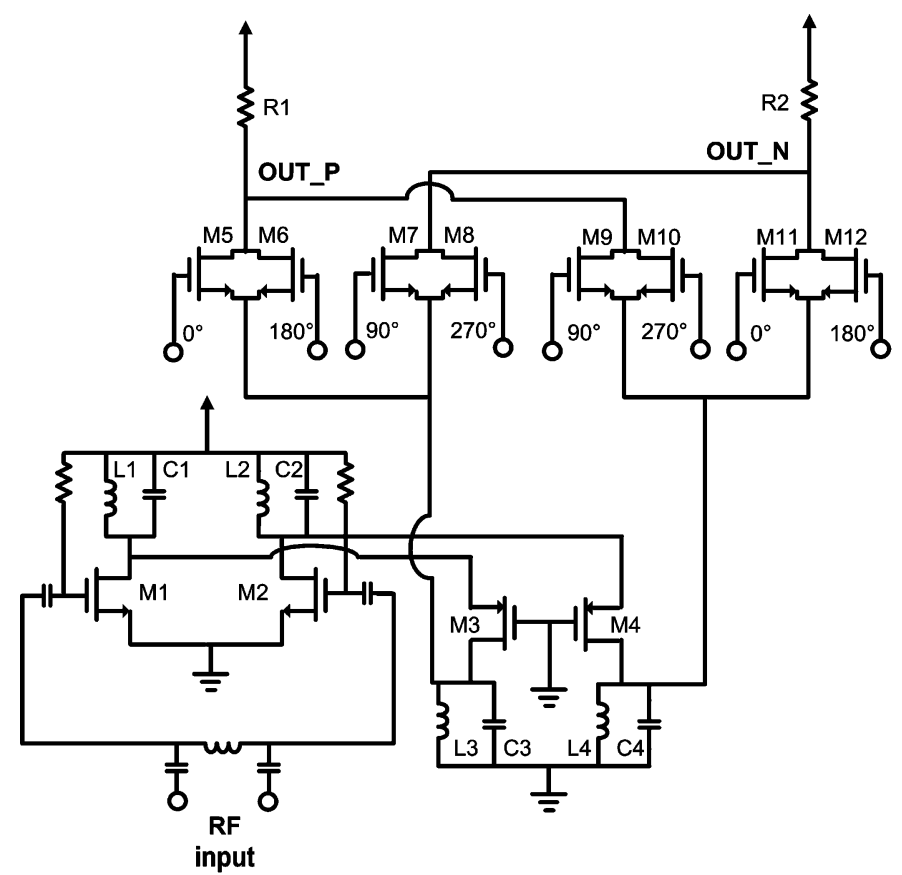

(a)

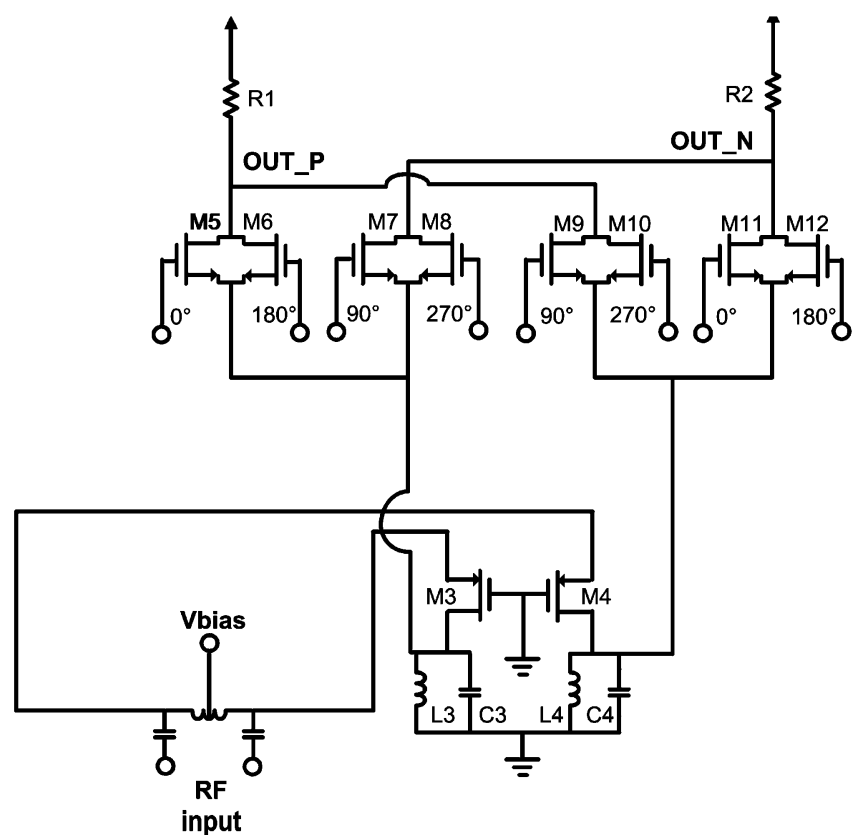

(b)

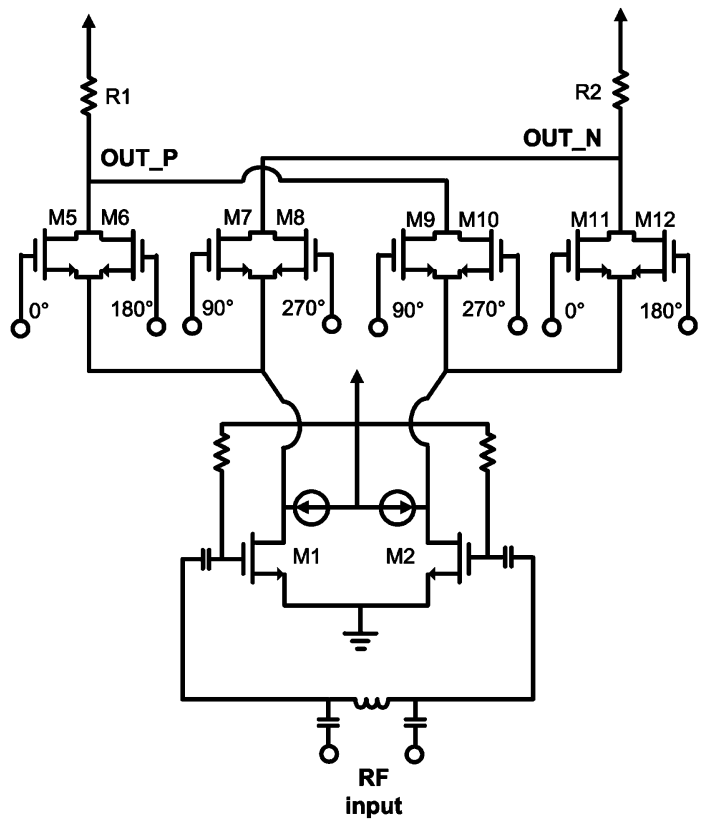

(c)

Fig. 3. Schematics of (a) the proposed subharmonic mixer, (b) the single-folded subharmonic mixer, and (c) the conventional nonfolded subharmonic mixer

with LO signals operating at one- $n$th of RF. Although the parallel-connected transistor pair switch configuration for SHMs of this work has been adopted in [9], there is still a difference between the SHMs in these two works. In this work, the transformer used as the gain-stage (transconductor) of the SHM in [9] has been modified to a $L C$-folded cascode amplifier for low voltage operation. This is because the performances of the SHM in [9] with a supply voltage of $1 \mathrm{~V}$ are inferior to those of the proposed SHM in this work in terms of noise figure, gain and linearity which will be reported in Section IV-B.

The $L C$ folded-cascode topology was previously applied to a LNA to achieve low-voltage operation and provide good re- verse isolation, but not to the transconductor stage of a mixer [10]. Therefore, the mixer in [10] would still suffer from LO self-mixing problem. In this work, the $L C$ folded-cascode is adopted in the transconductor stage of SHMs for the first time to obtain better LO-to-RF isolation. The schematic of the proposed SHM is shown in Fig. 3(a). The inductors (L1-L4) provide low resistance paths for DC bias currents of transistors (M1-M12). When the RF signal is present, the nMOS M1 and M2 will act as a differential transconductor, which is used to amplify the incoming RF signal. The drain current of M1 or M2 contains the DC current along with the amplified RF current signal. To prevent delivering RF signal to the supply, the tank circuits (L1//C1 
TABLE I

Simulation Results of the Proposed SHM, THE Single-Folded SHM, AND THE NONFOLDED SHM

\begin{tabular}{|c|c|c|c|}
\hline $\begin{array}{c}\text { Simulated } \\
\text { Performances }\end{array}$ & $\begin{array}{c}\text { Proposed } \\
\text { SHM in Fig.3(a) }\end{array}$ & $\begin{array}{c}\text { Single-folded } \\
\text { SHM in Fig.3(b) }\end{array}$ & $\begin{array}{c}\text { Non-folded } \\
\text { SHM in Fig.3(c) }\end{array}$ \\
\hline Power Supply & $1.0 \mathrm{~V}$ & $1.0 \mathrm{~V}$ & $1.0 \mathrm{~V}$ \\
\hline DC Current & $13.6 \mathrm{~mA}$ & $5.2 \mathrm{~mA}$ & $8.2 \mathrm{~mA}$ \\
\hline Voltage Gain & $13.5 \mathrm{~dB}$ & $-1.2 \mathrm{~dB}$ & $10.2 \mathrm{~dB}$ \\
\hline NF & $17.0 \mathrm{~dB}$ & $25.9 \mathrm{~dB}$ & $27.2 \mathrm{~dB}$ \\
\hline IIP3 & $-1.8 \mathrm{dBm}$ & $9.1 \mathrm{dBm}$ & $-9.2 \mathrm{dBm}$ \\
\hline $\begin{array}{c}\text { LO leakage } \\
\text { (10\% mismatch) }\end{array}$ & $-107.5 \mathrm{dBV}$ & $-101.6 \mathrm{dBV}$ & $-84.0 \mathrm{dBV}$ \\
\hline Required Area & $0.85 \times 1.29 \mathrm{~mm}^{2}$ & $0.85 \times 0.92 \mathrm{~mm}^{2}$ & $0.85 \times 0.55 \mathrm{~mm}^{2}$ \\
\hline
\end{tabular}

and $\mathrm{L} 2 / / \mathrm{C} 2$ ) are designed to resonate at the operation frequency, i.e., at this frequency, the tanks exhibit high impedances, forcing the RF current to be folded into the source terminals of the pMOS M3 and M4. Functioning as a differential unity gain current buffer, the common-gate transistors M3 and M4 can also provide good reverse isolation in the SHM. The output RF current of the differential $L C$-folded cascode transconductor is then fed to the tank circuits (L3//C 3 and L4//C4). Being resonant at the operation frequency as well, the tank circuits again force the RF signals to flow into the source terminals of the nMOS M5-M12. The poly-phase LO signals are applied to the gates of M5-M12 which act as switches and translate the RF signal to baseband signal.

It seems that a single-folded structure with pMOS as input transconductance devices (i.e., removing M1-M2, L1-L2, C1-C2, and applying RF inputs directly to M3 and M4 as shown in Fig. 3(b)) should function equally well at the same low supply voltage while requiring only two $L C$ tanks and lower current. Simulation shows the LO-to-RF isolation of such a structure would be worse than that of the proposed one by $5.9 \mathrm{~dB}$ if $10 \%$ mismatch in the parallel-connected switches occurs. (According to the $9-\mu \mathrm{m}^{2}$ gate drawn area and the $0.1-\mathrm{V}$ gate overdrive of the switches devices, the $3 \sigma$ current mismatches between each device should be $10 \%$ based on foundry book information.) As a more detailed comparison, the simulation results of proposed SHM and the one employing single-folded structure with only pMOS as input transconductance devices are listed in Table I. Indeed, the single-folded SHM consumes lower current (38.2\%), smaller die area $(71.3 \%)$ and it even exhibits better linearity. However, the corresponding gain and NF may hinder the receiver because of poor sensitivity.

The $L C$ double-folded cascode topology is apparently good for LO-RF isolation and low voltage operation. However, it requires four $L C$ tanks, which is quite expensive in terms of chip area, and consumes more current compared to conventional nonfolded structure [shown in Fig. 3(c)]. Moreover, for circuits containing more inductors and occupying larger chip area, the coupling through the silicon substrate should be critical. (Measured results show that the substrate coupling due to one on-chip spiral inductor is below $-44 \mathrm{~dB}$ at $5.4 \mathrm{GHz}$ when the distance from the center of the inductor is beyond $800 \mu \mathrm{m}$ and adding another inductor would enhance the point-to-point substrate coupling by 4-6 dB.) To elaborate on the comparison and tradeoff, the simulated results of a conventional nonfolded SHM are also listed in Table I. At the expense of significant degradation in noise figure and linearity, the conventional SHM exhibits fair conversion gain with lower power consumption $(60.3 \%)$ and less die-area $(42.6 \%)$.

LO-to-RF Isolation Enhancement: Providing good reverse isolation, the cascode topology simultaneously reduces the leakage amount of LO and the second harmonic (twice LO) appearing at RF port. This means not only the LO-to-RF isolation is improved but also the re-radiation problem is prevented. Moreover, it is worth mentioning that the adopted $L C$-folded cascode configuration can demonstrate better LO-to-RF isolation in a SHM than in a conventional mixer as the LO frequency is half the RF in the former. This mainly results from the bandpass characteristic of the $L C$ tank circuits. Being resonant at RF, the tank circuits exhibit high impedance only at RF band. For signals at frequencies far from the tank resonant frequency, all the tank circuits can be viewed as low resistance signal paths to voltage supply or to ground.

Due to the frequency distinction between RF and LO in SHMs, all the tank circuits are out-of-resonance at LO and can be viewed as low resistance signal paths by the LO leakage. On the way LO leakage traveling to the RF port in SHMs, most of the leakage signal will be delivered to either the voltage supply or ground. Consequently, the LO leakage appearing at the RF port will be greatly reduced, which is reflected in the remarkable LO-to-RF isolation (64.8 dB) obtained in the proposed SHM to be seen later. By contrast, conventional direct-conversion mixers cannot benefit from the bandpass characteristic of tank circuits for the frequencies of $\mathrm{LO}$ and $\mathrm{RF}$ are the same. To our knowledge, this is the first time that the $L C$-folded cascode technique is applied to SHMs to demonstrate such advantage, which certainly reveals the significance of our work.

The $L C$ folded cascode mixer assumes that the $L C$ tanks are resonated at $\mathrm{RF}$ (or $2 \mathrm{LO}$ ) frequency to achieve low leakage. In fact, the $\mathrm{Q}$ of the on-chip inductors should be high enough for the tanks to provide signal paths with relatively low impedance at LO frequency so that LO leakage appearing at RF port can be suppressed. The suppression would not be as effective and the linearity may be suffered if the assumption is not sustained. According to our simulation, the $\mathrm{Q}$ of those adopted on-chip inductors is 10.1 at $5.8 \mathrm{GHz}$ and the corresponding impedances of the tanks are $87.5 \mathrm{ohm}$ at $2.9 \mathrm{GHz}$ while the output impedances of the cascode transconductance devices are $692.6 \mathrm{ohm}$. Therefore, the ratio between the two impedances would be 7.9 which should be high enough to support the assumption. By assuming a $10 \%$ device mismatch in the parallel-connected switches, simulated LO-leakage suppression as function of the impedance ratio was shown in Fig. 4, where it was found that the LO-leakage suppression would be improved by $8.5 \mathrm{~dB}$ when the impedance ratio was increased from 1 to 8.

Input-Referred DC-Offset Due to LO Self-Mixing: The input referred DC-offset voltage due to LO self-mixing has been specified in [3] and the power level difference in decibels between this term and the given LO can be defined as LO self-mixing rejection. For direct-conversion receivers, the former reflects 


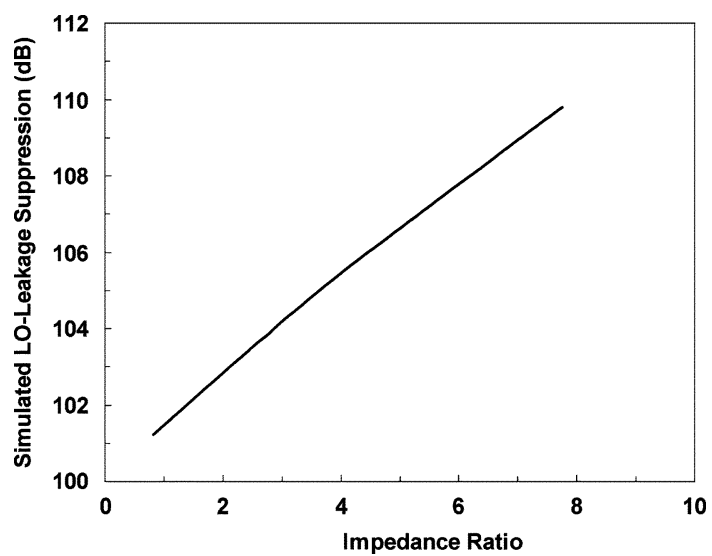

Fig. 4. The simulated LO-leakage suppression as function of impedance ratio between the cascode transconductor and the $L C$-tank.

SNR degradation due to LO self-mixing and the latter characterizes their capability to suppress LO self-mixing phenomenon. However, both issues were not fully addressed in most of the previously reported SHMs. LO self-mixing in mixers of direct-down conversion receivers should be reduced as much as possible since it leads to considerable DC-offset which seriously degrades the SNR of receivers. According to [3], the worst-case DC-offset due to LO self-mixing in mixers can be described as follows:

$$
\mathrm{V}_{\mathrm{DC}-\text { offset }}=\mathrm{V}_{\mathrm{LO}-\mathrm{LEA}} \cdot \mathrm{A}_{\mathrm{V}-\mathrm{LO}}
$$

where $\mathrm{V}_{\mathrm{LO}-\mathrm{LEA}}$ represents the observed LO leakage at the RF port and $A_{V-L O}$ stands for the conversion gain of a mixer when input signal at LO frequency is applied. This equation implies two ways to reduce the LO self-mixing DC-offset. One is reducing LO leakage, which can be realized by improving the LO-to-RF isolation. The other is reducing the conversion gain $A_{V-L O}$, which cannot improve the overall SNR if LO is equal to RF as in direct-down conversion receivers adopting conventional mixers. Since reducing conversion gain does not always improve SNR, a raw output offset voltage is inadequate for the measurement of LO self-mixing. Instead, a concept of input referred DC-offset voltage $V_{\text {IN-Referred }}$ introduced to evaluate the LO self-mixing is given by

$$
\mathrm{V}_{\mathrm{IN}-\text { Referred }}=\mathrm{V}_{\mathrm{LO}-\mathrm{LEA}}\left(\mathrm{A}_{\mathrm{V}-\mathrm{LO}} / \mathrm{A}_{\mathrm{V}-\mathrm{RF}}\right)
$$

where $A_{V-R F}$ represents the conversion gain of a mixer. The ratio $A_{V-R F} / A_{V-L O}$, defined as LOR in [5], stands for the rejection of incoming signal operating at LO frequency. Therefore, the total LO self-mixing rejection is equal to the summation of LO-to-RF isolation and LOR (in dB). For conventional direct-conversion mixers, $A_{V-L O}$ is equal to $A_{V-R F}$, namely the LOR is $0 \mathrm{~dB}$. Consequently, the overall rejection in LO selfmixing equals to the LO-to-RF isolation and $\mathrm{V}_{\mathrm{IN} \text {-Referred }}$ can be reduced only by minimizing LO leakage. In SHMs exhibiting huge loss in $\mathrm{A}_{\mathrm{V}-\mathrm{LO}}$, a considerable LOR can be achieved and counted in the LO self-mixing rejection. That is why SHMs can always exceed their conventional counterparts in LO selfmixing rejection by tens of $\mathrm{dB}$.

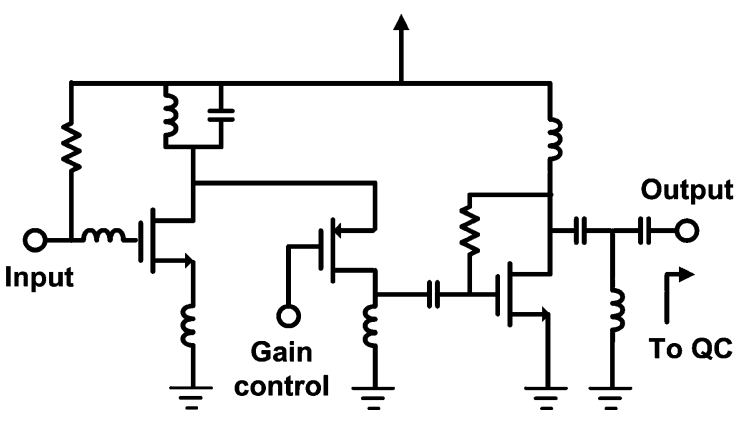

Fig. 5. Schematic of the low-noise amplifier.

\section{B. Low-Noise Amplifier}

The three-stage low-voltage LNA is depicted in Fig. 5. In the first stage, the cascode configuration is adopted to offer good isolation and frequency response. The $L C$-folded cascode topology in [10] is adopted in the LNA design to achieve low-voltage operation as well. With proper device selection for the input transistor and matching elements, broadband impedance matching and low-noise performance can be achieved simultaneously [11].

The main function of the LNA is to minimize the noise contribution of its following stages by providing a large power gain. Since the use of pMOS transistor inevitably suffers from smaller cut-off frequency, a common-source amplifier in the third stage is used to compensate for this penalty so that a better receiver SNR can be achieved. Note that this LNA is featured with gaincontrol that helps to provide a wider input dynamic-range and increase system efficiency. When the received signal strength is getting stronger, a low gain mode would be switched on by turning the common-gate pMOS off. In this way, the receiver power consumption could be lowered by $2 \mathrm{~mW}$. Therefore, the system battery lifetime can be lengthened, which is quite practical for the circuit to be integrated in hand-held devices or laptops with WiMAX 802.16e facilities.

\section{Quadrature Coupler}

As mentioned in Section II, a monolithic quadrature coupler (QC) is used to generate I/Q RF signals in the proposed receiver architecture. The adopted QC is actually a directional coupler implemented with lump elements as illustrated by the 4-port equivalent circuit in Fig. 6. How the terminals of the QC are actually connected to the LNA and the single-to-differential transformers is also depicted in Fig. 6. As a lump alternative of directional coupler, the QC can also be described in terms of $S$ parameters by the symmetrical matrix $S$ given by

$$
S=\frac{e^{j \alpha}}{\sqrt{2}}\left[\begin{array}{llll}
0 & 1 & 0 & j \\
1 & 0 & j & 0 \\
0 & j & 0 & 1 \\
j & 0 & 1 & 0
\end{array}\right] .
$$

The component values of the QC in Fig. 6 can be expressed as a function of the parameter $\alpha$ in (3) by the following relations:

$$
C 1=C 2=-\frac{1}{\omega Z_{0}}\left[\frac{1-\sqrt{2} \cos (\alpha)}{\cos (2 \alpha)}\right]
$$




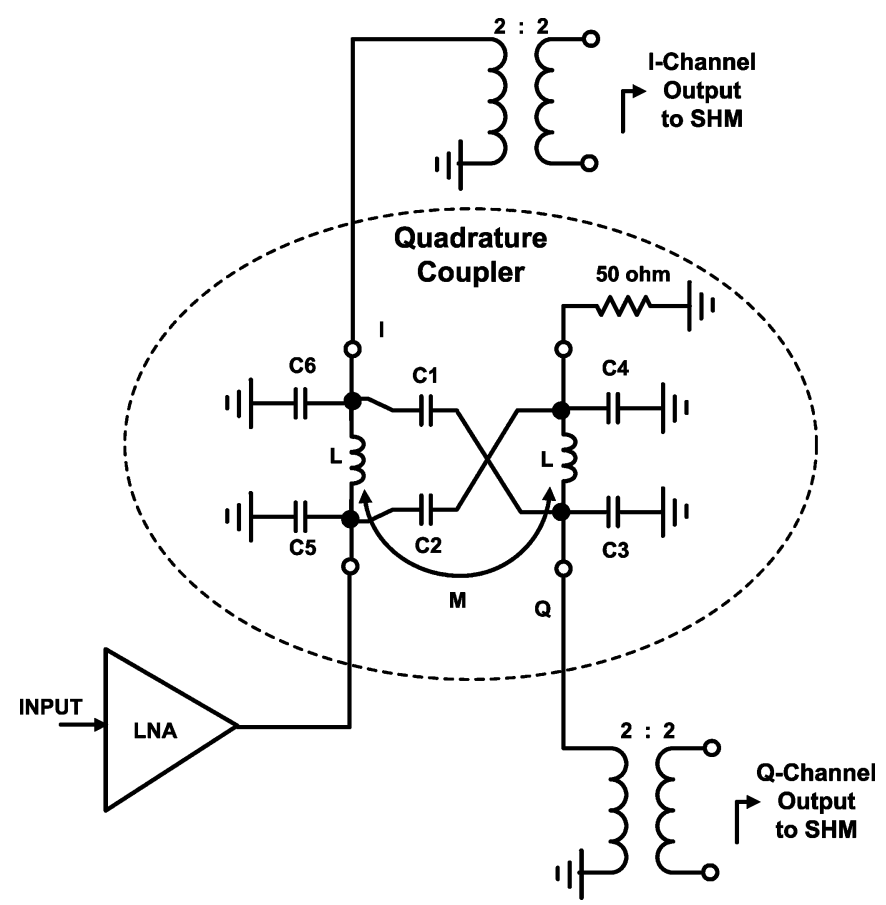

Fig. 6. Lump-element equivalent circuit of the on-chip quadrature coupler with its input terminal connected to the LNA and its quadrature output terminals connected to the single-to-differential transformers.

$$
\begin{aligned}
& C 3=C 4=C 5=C 6=\frac{1}{\omega Z_{0}}\left[-\frac{\sin (4 \alpha)}{1+\cos (4 \alpha)}\right. \\
&\left.\quad+\frac{1+\sqrt{2}(\sin (\alpha)-\cos (\alpha))}{\cos (2 \alpha)}\right] \\
& \frac{L}{M}=-\cot (\alpha) \\
& L=-\frac{1}{\omega} \frac{Z_{0} \sin (\alpha)}{\sqrt{2}}
\end{aligned}
$$

where $Z_{0}$ represents the characteristic impedance, $L$ is the inductance for each coil, $M$ represents the mutual inductance between the two coils, and $\omega$ represents the frequency of interest. Theoretically, the QC does not need to be designed with a characteristic impedance of $50 \Omega$, but choosing $50-\Omega$ impedance would facilitate on-wafer characterization. With $Z_{0}$ equal to $50 \Omega$, any choice of $\alpha$ would unequivocally determine C1-C6, $L$, and $M$. The corresponding component values for $\alpha=250^{\circ}-340^{\circ}$ were calculated and listed in Table II. The success of this design would be based on a right choice of $\alpha$ which can give us feasible component values. In this work, the optimal $\alpha$ value was chosen to be $300-310^{\circ}$ so that all the components could be easily realized.

According to [12], the inductors of the QC in Fig. 6 can be realized by two interleaved coils to make a compact design. Therefore, two 1.5-turn square spirals with a line width of $20-\mu \mathrm{m}$ were interleaved to form the main structure of the QC. Since the two interleaved inductors would certainly contain internal parasitic capacitances, the value of on-chip capacitors used to realize C1-C6 can be reduced. In particular, the physical structure of the inductors are designed in a way that the capacitors
TABLE II

COMPONENT VALUES OF THE QUADRATURE COUPLER FOR $\alpha=250^{\circ}-340^{\circ}$

\begin{tabular}{|c|c|c|c|c|c|}
\hline $\begin{array}{c}\text { Component } \\
\text { (unit) }\end{array}$ & $\begin{array}{c}\alpha \\
\text { (deg) }\end{array}$ & $\begin{array}{c}\mathrm{C} 1-\mathrm{C} 2 \\
\text { (fF) }\end{array}$ & $\begin{array}{c}\mathrm{C} 3-\mathrm{C6} \\
\text { (fF) }\end{array}$ & $\begin{array}{c}\mathrm{L} \\
(\mathrm{nH})\end{array}$ & $\begin{array}{c}\mathrm{M} \\
(\mathrm{nH})\end{array}$ \\
\hline \multirow{5}{*}{ Value } & 250.0 & 1044.9 & 343.7 & 0.9 & -0.3 \\
\cline { 2 - 6 } & 260.0 & 715.1 & 280.9 & 0.9 & -0.2 \\
\cline { 2 - 6 } & 270.0 & 539.5 & 223.5 & 1.0 & 0.0 \\
\cline { 2 - 6 } & 280.0 & 433.1 & 170.1 & 0.9 & 0.2 \\
\cline { 2 - 7 } & 290.0 & 363.6 & 119.6 & 0.9 & 0.3 \\
\cline { 2 - 7 } & 300.0 & 316.0 & 71.0 & 0.8 & 0.5 \\
\cline { 2 - 7 } & 310.0 & 282.6 & 23.6 & 0.7 & 0.6 \\
\cline { 2 - 7 } & 320.0 & 259.0 & -23.6 & 0.6 & 0.7 \\
\cline { 2 - 7 } & 330.0 & 242.5 & -71.0 & 0.5 & 0.8 \\
\cline { 2 - 7 } & 340.0 & 231.7 & -119.6 & 0.3 & 0.9 \\
\hline
\end{tabular}

(C3-C6) between each port and ground can be fully realized by the internal parasitic capacitances of inductors and the on-chip capacitors used to realize C1 (and C2) is reduced to $284 \mathrm{fF}$.

An electromagnetic (EM) simulation was performed to fully characterize the QC. It is found that the frequency response in either phase or magnitude is almost independent of the source impedance. In particular, the forward power gain of the coupler is insensitive to the impedance mismatch at each port of the device, which greatly differs from the situation of a band-selection filter ahead of a receiver or an image reject filter used in a heterodyne receiver and makes the QC a reliable on-chip element.

\section{Transformers and Poly-Phase Filters (PPFs)}

On-chip transformers were designed for single-to-differential conversion in RF/LO signal path. As shown in Fig. 6, each output signal of the QC would be respectively converted into a pair of differential signals by a transformer before entering the SHM. Note that this 2:2 transformer should be considered as part of the inter-matching network from the coupler to SHMs in order to achieve precise phase shift and balance magnitude in I/Q output signals. In addition, the operating frequency should be optimized at $5-\mathrm{GHz}$ band to prevent serious SNR degradation. Another transformer with a turn-ratio of 4:4 was used to generate differential LO from the external single-ended signal source for measurement convenience. The line width of the coils in the transformers is $6 \mu \mathrm{m}$ and the line space is $2 \mu \mathrm{m}$. For all the transformers, the primary coils are made of $2-\mu \mathrm{m}$-thick aluminum (M6) while the secondary ones are made of $0.8-\mu \mathrm{m}$-thick aluminum. To achieve a maximum power transfer, this transformer not only offers single-to-differential conversion in LO but also functions as a matching element to translate the differential input impedance of the PPF into 50 ohm. Simulation was performed on the PPF by using RF models of resistors and capacitors provided by UMC $0.18-\mu \mathrm{m}$ CMOS device library and assuming that all the devices are perfectly matched. At $2.7 \mathrm{GHz}$, the loss of PPF was $14.6 \mathrm{~dB}$ while the quadrature imbalances are less than $5^{\circ}$ within a $500-\mathrm{MHz}$ band from 2.5 to $3.0 \mathrm{GHz}$. The simulation considering mismatches between resistors (capacitors) was also performed. It was found the resistor mismatches affect the amplitude imbalance more while the capacitor mismatches affect the phase error more. At 2.9-GHz, magnitude imbalances of $0.02 \mathrm{~dB}$ 
$(0.004 \mathrm{~dB})$ and $0.16 \mathrm{~dB}(0.056 \mathrm{~dB})$ along with phase errors of $1.4^{\circ}\left(1.5^{\circ}\right)$ and $1.87^{\circ}\left(0.39^{\circ}\right)$ can be observed if mismatches of $1 \%$ and $10 \%$ between resistors (capacitors) were assumed, respectively. According to the simulation, the quadrature phase errors between LO signals would be translated into the phase errors between differential output signals of each SHM while the amplitude mismatches have minor influence on the output waveform.

\section{MeAsured Results AND Discussion}

\section{A. Quadrature Coupler and Transformers}

Since the quadrature coupler (QC) and the transformers are placed in the RF paths, their performance in terms of loss and phase matching would be critical to the receiver's performance. Simulations and measurements were performed on the standalone QC. The simulation results were obtained from the layout view of the $\mathrm{QC}$ by using a 2.5-D electromagnetic simulator (Agilent ADS momentum) given with process parameters of UMC 0.18- $\mu \mathrm{m}$ CMOS technology. From 5.7 to $5.9 \mathrm{GHz}$, the QC exhibits a phase error less than $0.06^{\circ}$ [Fig. 7(a)] and a magnitude error below $0.6 \mathrm{~dB}$ [Fig. 7(b)] in the simulation while its loss is below $3.9 \mathrm{~dB}$ [Fig. 7(c)]. The simulated side-band rejection is shown in Fig. 7(d) where rejections of 29.2, 35.4, and $60.1 \mathrm{~dB}$ are achieved at 5.7, 5.8, and $5.9 \mathrm{GHz}$, respectively. Since the $\mathrm{QC}$ is a passive element, its loss also represents its noise figure. According to simulation, the system noise figure would be degraded by $1.09 \mathrm{~dB}$ when the QC was inserted between LNA and mixers.

The statistical measurement results from three samples were shown together with the simulation results in Fig. 7(a)-(d) for comparison. In general, the curves obtained from the measurements follow closely with those generated by electromagnetic simulation. However, some differences between them can still be observed. From 5.7 to $5.9 \mathrm{GHz}$, the measured phase errors were $1.3-4.8^{\circ}$ [Fig. 7(a)], the measured magnitude errors were $0.7-2.5 \mathrm{~dB}$ [Fig. 7(b)] and the measured losses were 3.6-4.2 dB [Fig. 7(c)]. The achieved side-band rejections were 17.0-24.1 dB [Fig. 7(d)] which were apparently worse than the simulation results. From Fig. 7(d), it can be found the optimal operating frequency of the QC was shifted from $5.9 \mathrm{GHz}$ to 6.2-6.5 GHz.

Mismatches of $\pm 10 \%$ and $\pm 15 \%$ between $\mathrm{C} 1$ and $\mathrm{C} 2$ are considered respectively as shown in Fig. 7(e). According to the simulated side-band rejection shown in Fig. 7(e), the sideband rejection at $5.9 \mathrm{GHz}$ would be degraded from $60.1 \mathrm{~dB}$ to $54.4 \mathrm{~dB}$ and $46.8 \mathrm{~dB}$, respectively, when the worse-case mismatches of $\pm 10 \%$ and $\pm 15 \%$ occurred. Furthermore, the IQ imbalance degradation due to process variations were also investigated as shown in Fig. 7(f). It was found the side-band rejection at $5.9 \mathrm{GHz}$ would be degraded from $60.1 \mathrm{~dB}$ to $22.4 \mathrm{~dB}$ and $16.1 \mathrm{~dB}$, respectively, when the variations of $+15 \%$ and $+30 \%$ occurred. Meanwhile, the optimal frequency was shifted to $5.6 \mathrm{GHz}$ and $5.2 \mathrm{GHz}$, respectively.

Since the QC is designed by employing the inductor parasitic as capacitors, the resulting frequency response could vary significantly as the parasitic cannot be properly controlled. Besides, if a wider frequency range from 5 to $6 \mathrm{GHz}$ is considered, the magnitude error can be as high as $4 \mathrm{~dB}$. Therefore, I/Q mismatch compensation would be required for receivers using such on-chip coupler in practical realization. There are several ways to implement mismatch compensation. For example, load resistors in mixers can be made switchable so that the amplitudes of I and Q channels can be controlled by control words [13]; computing resources of digital companion chips or test tone generation along with internal power detection can also be used to eliminate I/Q mismatch automatically [14]-[16].

A stand-alone transformer with a turn ratio of $2: 2$ was fabricated for on-chip measurement and characterization. Both the primary coil and secondary coil have one of their terminals grounded so that the transformer could be measured as a twoport element. According to the obtained $S$-parameters, the minimum loss of the transformer is from 2.3 to $3.3 \mathrm{~dB}$ over the band of interest.

\section{B. Low-Voltage Subharmonic Mixer}

A stand-alone test-circuit of the proposed SHM was realized in the $0.18-\mu \mathrm{m}$ CMOS technology to show its advantage in LO self-mixing suppression. Drawing $13.6 \mathrm{~mA}$ from a $1-\mathrm{V}$ voltage supply, the circuit achieves a voltage gain of $12.9 \mathrm{~dB}$ and a NF of $17.3 \mathrm{~dB}$ at $5-\mathrm{GHz}$ band. The measured performances of the proposed SHM along with the one in [9] were summarized and compared in Table III. The linearity of the SHM in [9] strongly depends on the supply voltage and therefore its linearity would be degraded when the supply voltage is reduced to $1-\mathrm{V}$. Under the constraint of $1-\mathrm{V}$ operation, the performances of proposed SHM are better than those of the one in [9] except for power consumption.

According to (2), the worst-case input-referred DC-offset would equal to the LO leakage divided by the LOR. Therefore, to obtain such an input-referred DC-offset, LO leakage at RF port and the LOR need to be measured first. As mentioned in Section III, the LO self-mixing rejection is defined as the power difference in decibel between the given LO signal and the input-referred LO-self-mixing induced DC-offset. Once the LO leakage and LOR are measured, along with the known LO input level, the least achievable LO self-mixing rejection can be obtained as follows:

$$
\begin{aligned}
& \text { LO self - mixing rejection }(\mathrm{dB}) \\
& =\mathrm{LO} \text { input }(\mathrm{dBm})-\text { input-referred } \mathrm{DC}-\text { offset }(\mathrm{dBm}) \\
& =\mathrm{LO} \text { input }(\mathrm{dBm})-[\mathrm{LO} \text { leakage }(\mathrm{dBm})-\mathrm{LOR}(\mathrm{dB})] \\
& =\mathrm{LO}-\mathrm{to}-\mathrm{RF} \text { isolation }(\mathrm{dB})+\mathrm{LOR}(\mathrm{dB})
\end{aligned}
$$

An external LO power of $15.5 \mathrm{dBm}$ was applied during the whole measurement. The LO signal required to drive the SHM was provided by the signal generator and therefore the $2 \times \mathrm{LO}$ component at $\mathrm{LO}$ port was very low $(-86.9 \mathrm{dBc})$. According to simulation, the loss due to on-chip transformer and poly-phase filter is $15.9 \mathrm{~dB}$. Therefore, the output power would be $-0.4 \mathrm{dBm}$ for each of the poly-phase LO signals pumping the switches. An LO leakage of $-65.2 \mathrm{dBm}$ can be observed at the RF port and the measured LOR is $35.5 \mathrm{~dB}$. A remarkable LO-to-RF isolation $(64.8 \mathrm{~dB})$ is demonstrated as a result of the cascode configuration along with additional low resistance 


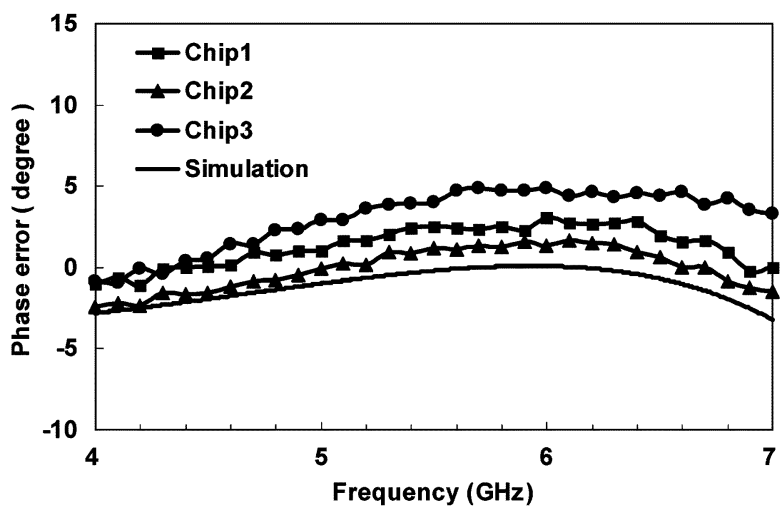

(a)

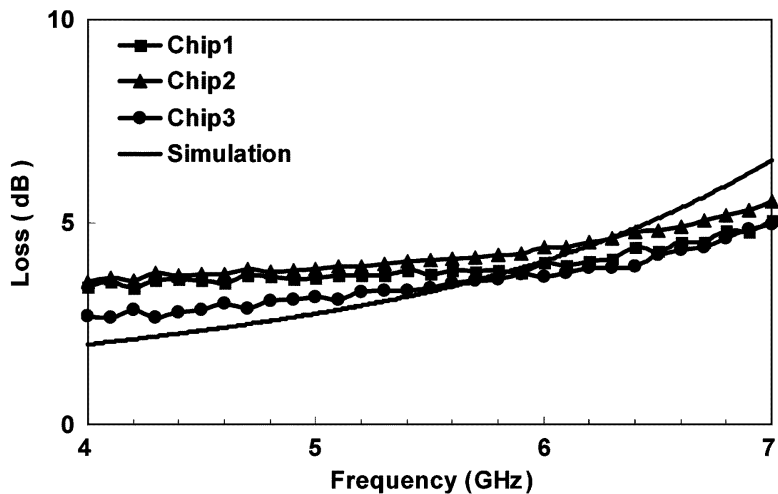

(c)

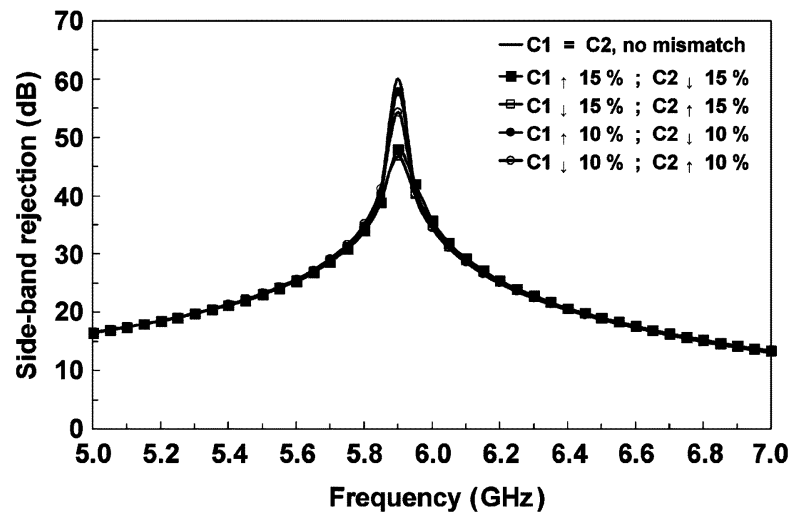

(e)

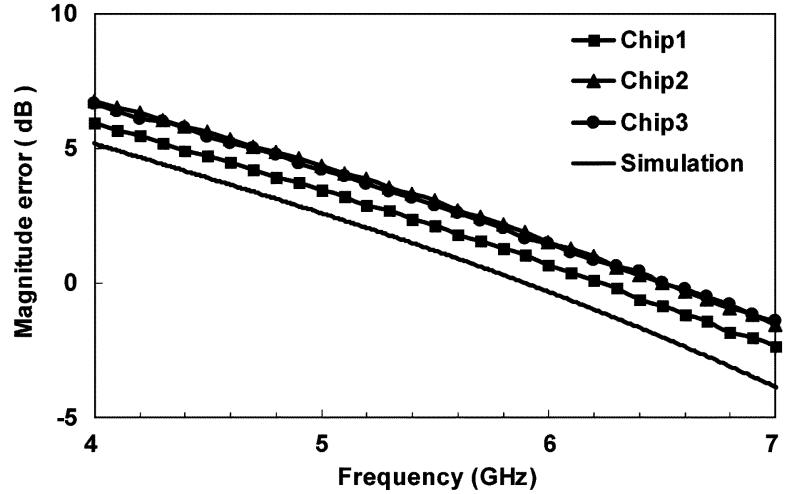

(b)

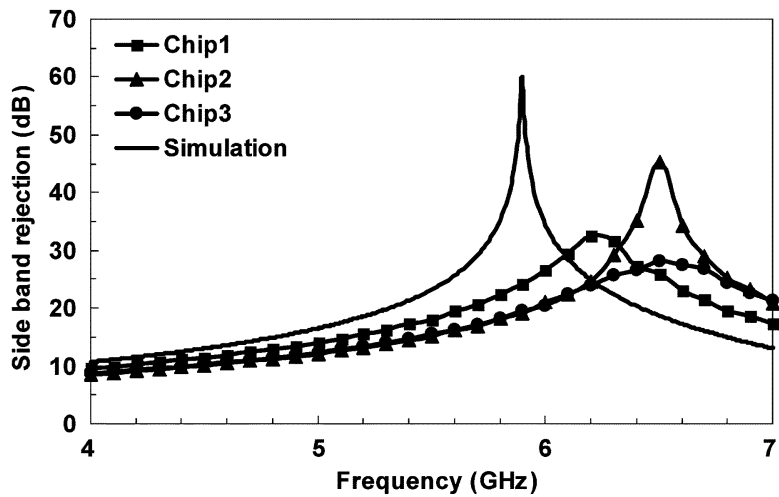

(d)

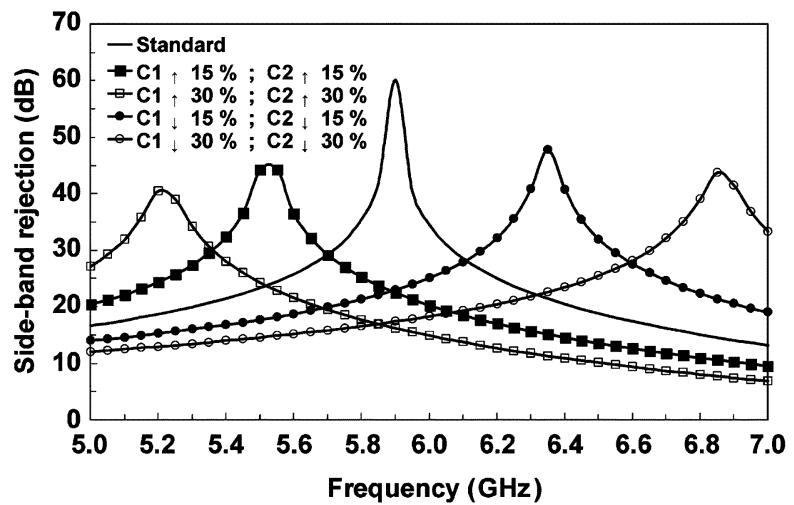

(f)

Fig. 7. Measured and simulated (a) phase error, (b) magnitude error, (c) loss, and (d) side-band rejection of the quadrature coupler. Side-band rejection simulation of the quadrature coupler with component (e) mismatches and (f) process variation.

TABLE III

MEASUREMENT RESUlTS OF PROPOSED SHM AND SHM IN [9]

\begin{tabular}{|c|c|c|c|}
\hline Measured Results & Proposed SHM & \multicolumn{2}{|c|}{ SHM in [9] } \\
\hline Supply (V) & 1.0 & 1.0 & 1.8 \\
\hline Power (mW) & 13.6 & 1.8 & 3.5 \\
\hline Gain (dB) & 12.9 & 8.2 & 10.8 \\
\hline NF (dB) & 17.3 & 22.7 & 25.5 \\
\hline IIP3 (dBm) & 2.8 & 2.3 & 7.9 \\
\hline
\end{tabular}

signal paths provided by the tank circuits for out-of-resonance signals. Consequently, for this SHM, the input-referred DC-offset due to the LO self-mixing is $-100.7 \mathrm{dBm}$ and the rejection of LO self-mixing is $100.3 \mathrm{~dB}(=64.8 \mathrm{~dB}+35.5 \mathrm{~dB})$. The circuit performance greatly exceeds the state of the art. In addition, a very low $2 \times \mathrm{LO}$ leakage $(-101.9 \mathrm{dBm})$ was observed at the RF port, which would favor constituted receivers with re-radiation immunity. Note that although the $2 \times \mathrm{LO}$ component at the LO input is very small $(-86.9 \mathrm{dBc})$, it would be generated by the switching-function in SHMs and appear appreciably at RF port if the isolation of SHMs is not good enough as evidenced by the reported data in [5]-[7] (see Table IV). All the performances of the proposed SHM and some recently reported SHMs [3]-[7] are summarized in Table IV. Powered by the lowest operation voltage $(1 \mathrm{~V})$, the proposed SHM demonstrates the lowest input referred DC-offset among the reported SHMs while its other performances are comparable with or even better than those of the previous works.

\section{Receiver Front-End}

A receiver front-end with I/Q channels is implemented by using the proposed low-voltage SHMs, LNA, transformers and 
TABLE IV

PERFORMANCE SUMMARY OF THE SHMs

\begin{tabular}{|c|c|c|c|c|c|c|}
\hline Items & $\begin{array}{c}\text { This } \\
\text { Work }\end{array}$ & {$[3]$} & {$[4]$} & {$[5]$} & {$[6]$} & {$[7]$} \\
\hline Technology & CMOS & BiCMOS & CMOS & SiGe & SiGe & SiGe \\
\hline Supply (V) & 1.0 & 2.7 & 3.0 & 3.3 & 3.3 & 3.0 \\
\hline Power (mW) & 13.6 & N.A. & 5.2 & 9.2 & 16.5 & 12.6 \\
\hline Freq (GHz) & 5.2 & 1.9 & 0.9 & 2 & 5.2 & 2.1 \\
\hline Gain (dB) & 12.9 & 27.0 & 13.0 & 17.2 & 6 & 18.0 \\
\hline NF (dB) & 17.3 & 14.0 & 24.5 & 9.8 & N.A. & 8.0 \\
\hline IIP3 (dBm) & 2.8 & -4.0 & -10.6 & -5.1 & 4.6 & -6.6 \\
\hline IIP2 (dBm) & 45.9 & 37.0 & 35.7 & 31.7 & 29.0 & 27.0 \\
\hline LOR (dB) & 35.5 & N.A. & 37.5 & 35.0 & N.A. & N.A. \\
\hline LO leakage (dBm) & -65.2 & N.A. & $-59.4 \dagger$ & N.A. & -55 & -44.0 \\
\hline 2LO leakage (dBm) & -101.9 & N.A. & N.A. & -61.8 & -60 & -44.0 \\
\hline DC-Offset (dBm) & -100.7 & -97.0 & $-96.9 \dagger$ & N.A. & N.A. & N.A. \\
\hline
\end{tabular}

t: $50 \mathrm{~dB}$ LO-to-RF isolation is assumed.

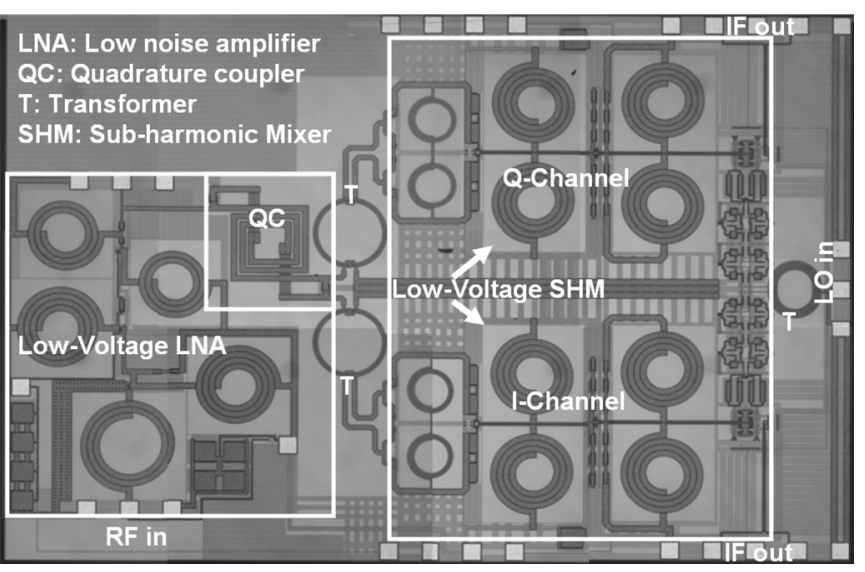

Fig. 8. Die photo of the realized receiver front-end.

QC. The die photo is shown in Fig. 8 and the die area is $2.98 \times$ $2.03 \mathrm{~mm}^{2}$. The on-wafer probing measurement was performed to prevent side issues from wire-bonding or packaging. Inductor $\mathrm{Q}$ factor degradation at high frequencies due to silicon substrate loss is a well-known weakness of CMOS technology. In addition, as mentioned previously, for circuits containing more inductors and occupying larger chip area, the coupling through the silicon substrate should be critical [17]. Therefore, another set of experimental results was taken from a chip applied with CMOS compatible inductively coupled plasma (ICP) to selectively remove the substrate under all the inductors/transformers [11].

The circuit consumes $45.5 \mathrm{~mW}$ at $1.0-\mathrm{V}$ operation. Firstly, the voltage gain of the realized receiver front-end was measured with different on-chip LO power used to drive SHMs. As shown in Fig. 9(a), the voltage gain reached its peak value $(25.2 \mathrm{~dB})$ when an on-chip LO power of $14.6 \mathrm{dBm}$ was provided. There- fore, we adopted this LO level to proceed the following measurements. Note that, for $1-\mathrm{V}$ operation, the on-chip LO power could not be as large as $14.6 \mathrm{dBm}$. Performances at lower and more practical LO signal levels were therefore obtained from simulation as show in Fig. 9(b). It can be found the gain would be decreased by $7.3 \mathrm{~dB}$ and the noise figure would be degraded by $1.2 \mathrm{~dB}$ when a $\mathrm{LO}$ signal of $4 \mathrm{dBm}$ is applied.

The $\mathrm{S}_{11}$ of the front-end (before and after the ICP process) was shown in Fig. 10. Clearly, the measured $\mathrm{S}_{11}$ fell down below $-10 \mathrm{~dB}$ over the band of interest $(5-6 \mathrm{GHz})$, indicating that a wideband impedance matching was obtained by the useful methodology of LNA device selection proposed in [11]. It was found that the optimal input match frequency (the dip of the $S_{11}$ curve) was shifted from $5.1 \mathrm{GHz}$ to $5.3 \mathrm{GHz}$ due to the substrate parasitic reduction by ICP post process.

To verify the RF quadrature generation achieved by the integrated QC and the single-to-differential conversion by on-chip transformers, time-domain output waveforms of down-converted signals were measured, as shown in Fig. 11(a) and (b). For measurement convenience, the following measurements were performed only on one of the I/Q branches.

The noise figure of the whole receiver at $5.4 \mathrm{GHz}$ was measured and shown in Fig. 12. With silicon removal underneath the inductors and transformers, the circuit exhibited a spot noise figure of $5.2 \mathrm{~dB}$ when a signal at $5.420 \mathrm{GHz}$ was down converted to $20 \mathrm{MHz}$. Even without silicon removal, the receiver still shows a reasonably good NF of $7.0 \mathrm{~dB}$. The $1 / \mathrm{f}$ noise of this circuit with a corner frequency of $3-3.5 \mathrm{MHz}$ would be a problem for some communication systems. To cope with this issue, further careful design techniques such as increasing transistor size [18], [19] or reducing the bias current [20] of the SHM switches can be used to lower the corner frequency of $1 / f$ noise. In addition, any circuit mismatch should be minimized, because 1/f noise from transconductor transistors may appear at the output of SHMs due to RF-IF feed-through [18]. 


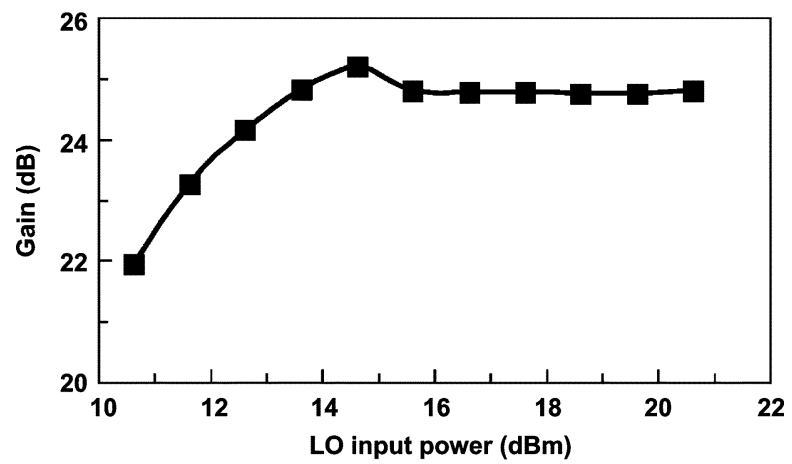

(a)

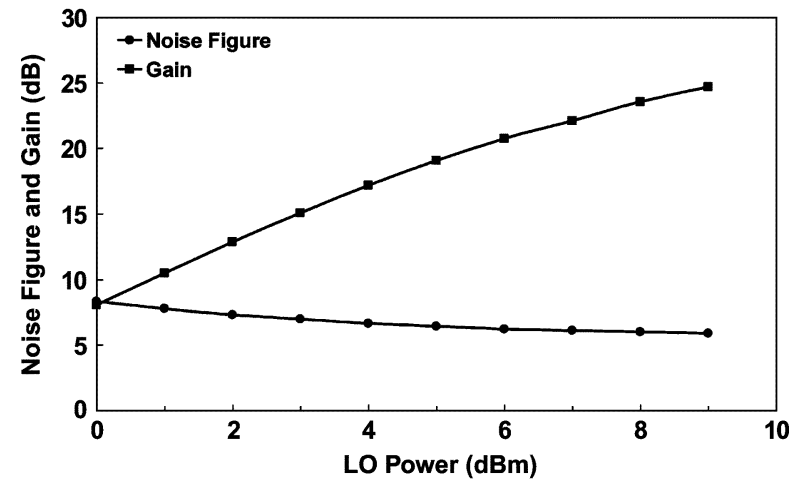

(b)

Fig. 9. (a) Measured voltage gain of the proposed receiver front-end under different LO power levels. (b) Simulated voltage gain and noise figure of the proposed receiver front-end under lower LO power levels.

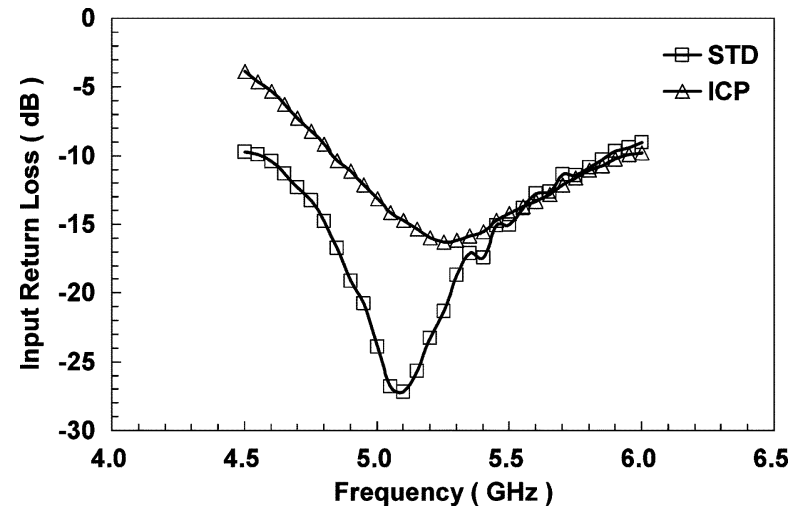

Fig. 10. Measured $\mathrm{S}_{11}$ of the proposed receiver front-end (before and after ICP post process).

As the most important characteristic of down-converters utilizing SHMs, the LOR was also measured. As shown in Fig. 13, a LOR of $54.9 \mathrm{~dB}$ can be achieved at $5.4 \mathrm{GHz}$ even without the ICP post-IC process. Theoretically, LOR depends mainly on circuits' physical symmetry and the ICP post process should have been irrelevant to it. However, the proposed SHMs can benefit from the bandpass characteristic of constituting tank circuits in achieving good LOR as well since the Q-factor of the tank circuits will be improved due to substrate-loss reduction by ICP. Therefore, it is reasonable the LOR can be enhanced after the ICP post process as shown in Fig. 13.

To demonstrate the advantage of adopting SHM in a direct-conversion receiver, the input-referred DC-offset due to LO self-mixing of the realized circuit was calculated with the

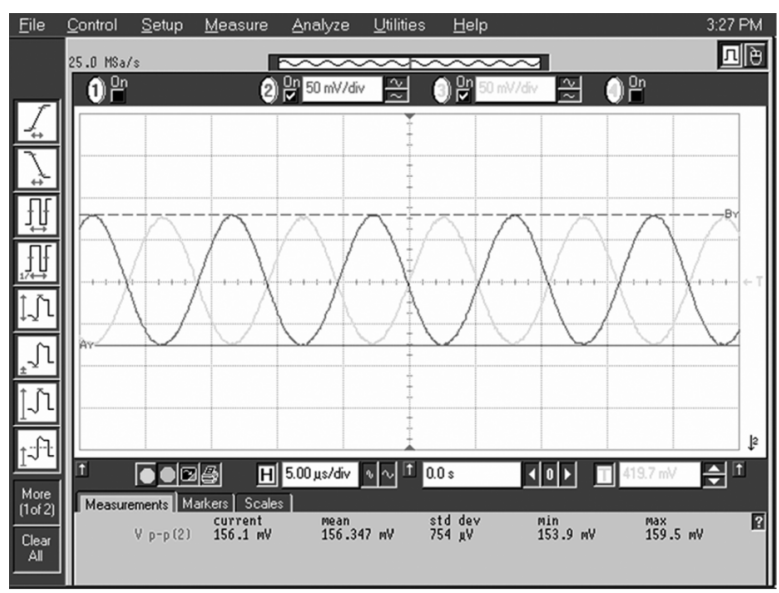

(a)

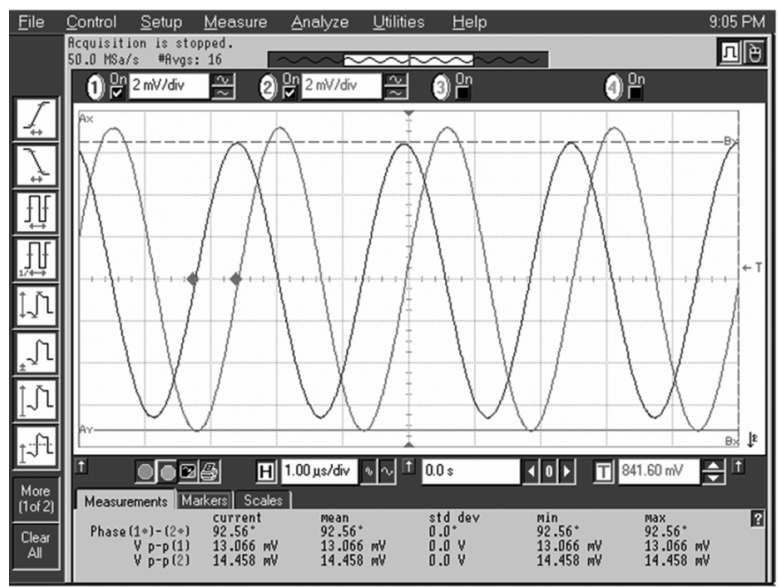

(b)

Fig. 11. Measured time-domain (a) differential (b) quadrature output waveforms.

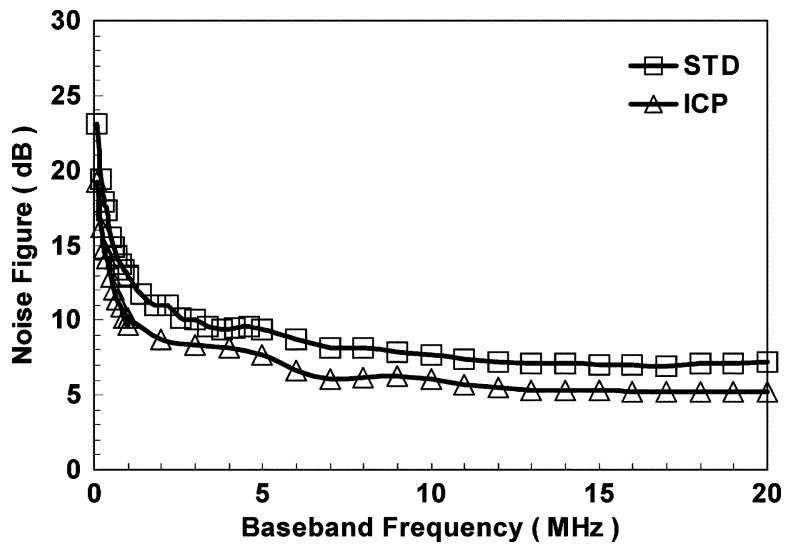

Fig. 12. Measured noise figure of the proposed receiver front-end (before and after ICP post process).

LO leakage at both the inputs of LNA and SHMs taken into account. The calculated input-referred DC-offset due to LO self-mixing was $-110.7 \mathrm{dBm}$ which was below the noise floor at the input of receiver $(-107.1 \mathrm{dBm}$ at $5-\mathrm{MHz}$ bandwidth) and the proposed receiver front-end can be regarded as a LO self-mixing-free circuit.

The measured frequency spectra showing the LO and 2LO leakage at the RF port were presented in Fig. 14(a) and (b), 


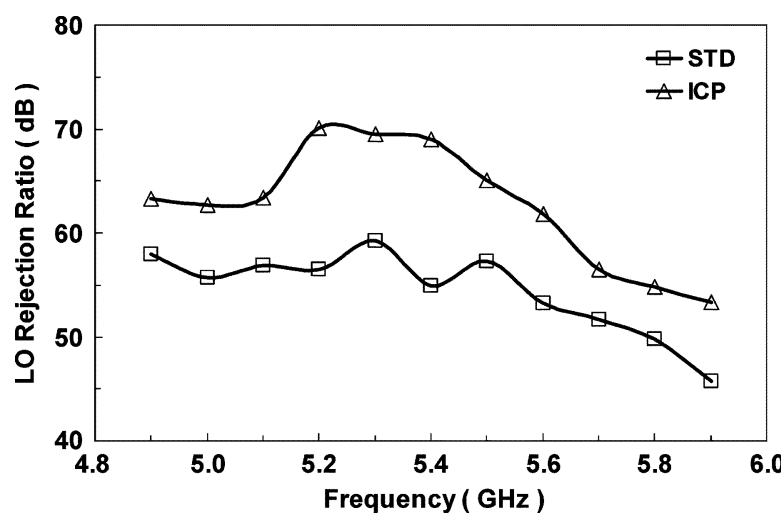

Fig. 13. Measured LO rejection ratio of the proposed receiver front-end (before and after ICP post process).

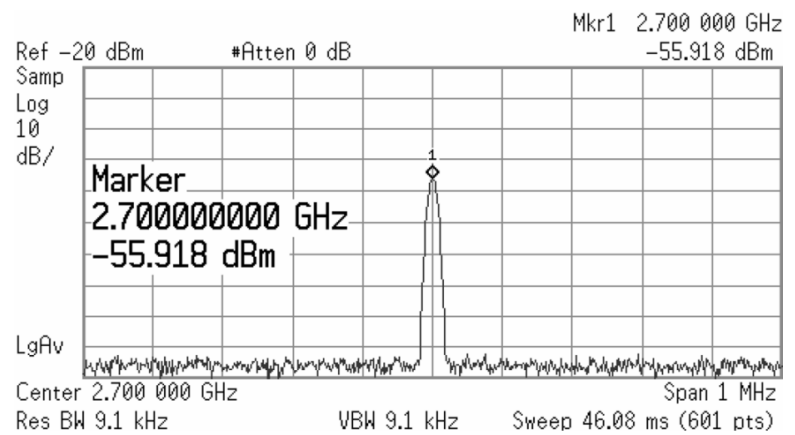

(a)

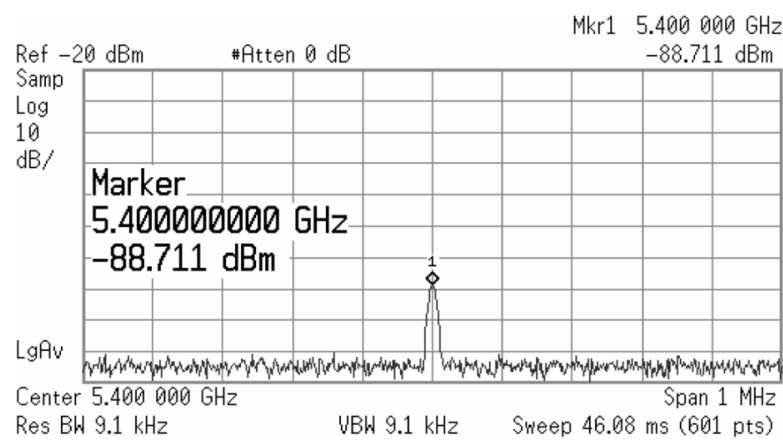

(b)

Fig. 14. Measured frequency spectra showing (a) the LO leakage and (b) the 2LO leakage at the RF port.

respectively. Based on the method used in [3], a direct measurement of LO self-mixing input-referred DC-offset was performed. The measured input-referred DC-offsets under different LO input levels are shown in Fig. 15. The results show that the input referred DC-offset due to the LO self-mixing is within $2 \mu \mathrm{V}$ in the proposed circuit.

The results of the two-tone test and the gain-compression versus input power were shown in the Fig. 16 and Fig. 17, where IIP3 is $-12.5 \mathrm{dBm}$ and the P1 dB is $-21.2 \mathrm{dBm}$. Since the maximum signal handled by the receivers is $-30 \mathrm{dBm}$ for both WLAN 802.11a and WiMAX standards, the P1 dB of a receiver should be at least $-26 \mathrm{dBm}$ and the required IIP3 should be larger than $-16 \mathrm{dBm}$. The receiver linearity was estimated by system simulator given with the performances of the proposed front-end in terms of IIP3. It was found the receiver could

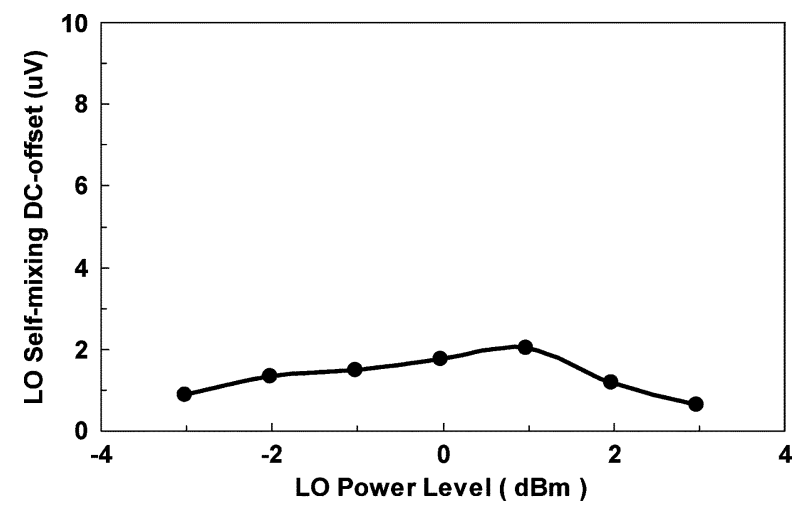

Fig. 15. Measured input referred DC-offset due to LO self-mixing.

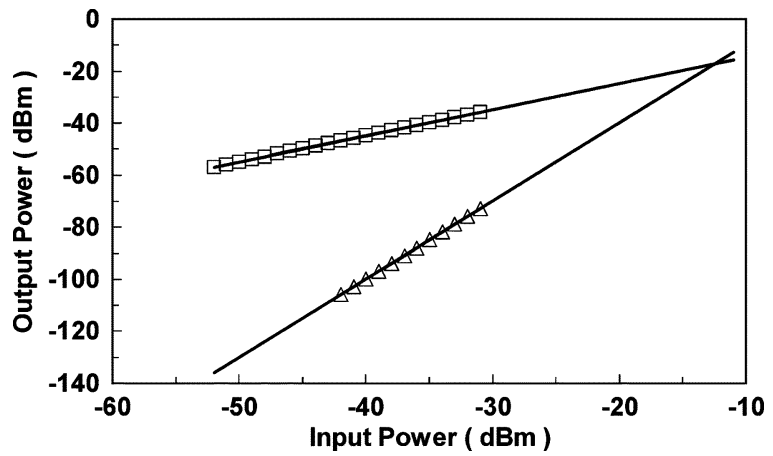

Fig. 16. The output level of fundamental and third-order inter-modulation in a two-tone test for measuring IIP3.

achieve an IIP3 of $-15.9 \mathrm{dBm}$ while adopting the proposed front-end followed by an analog baseband with 12-dBm IIP3 which is normally achievable. All the measured performances at $5.4 \mathrm{GHz}$ were summarized in Table V. According to these experimental results, it is promising to apply the proposed RF front-end architecture to direct-conversion receivers for WLAN 802.11a or WiMAX 802.16e.

\section{CONCLUSION}

For direct conversion receivers, $\mathrm{RF}$ front-end circuits clear of LO self-mixing are always desirable. In addition, low voltage operation is essential for system integration in deep-submicron CMOS technology. Therefore, $L C$-folded-cascode configuration is utilized in LNA and SHMs to simultaneously achieve low-voltage operation and high LO self-mixing rejection in this work. For IQ generation at IF port of the SHMs, a quadrature coupler is also designed and integrated. A $4.9-5.9-\mathrm{GHz}$ monolithic receiver front-end constituted by these building blocks was fabricated in $0.18-\mu \mathrm{m}$ CMOS process. According to the measurement results, the realized circuit well suits the 5-GHz range applications. The input return loss of the circuit is well below $-10 \mathrm{~dB}$ from $4.9 \mathrm{GHz}$ to $5.9 \mathrm{GHz}$. In spite of more than one on-chip passive elements along the RF signal path, this circuit exhibits high voltage gain and fair NF as a result of interface impedance matching from LNA to QC, the transformers and then the SHMs. Drawing $45.5 \mathrm{~mA}$ from a $1-\mathrm{V}$ voltage supply, this circuit achieves a voltage gain of $26.2 \mathrm{~dB}$ at $5.4 \mathrm{GHz}$. The measured spot noise figure at 


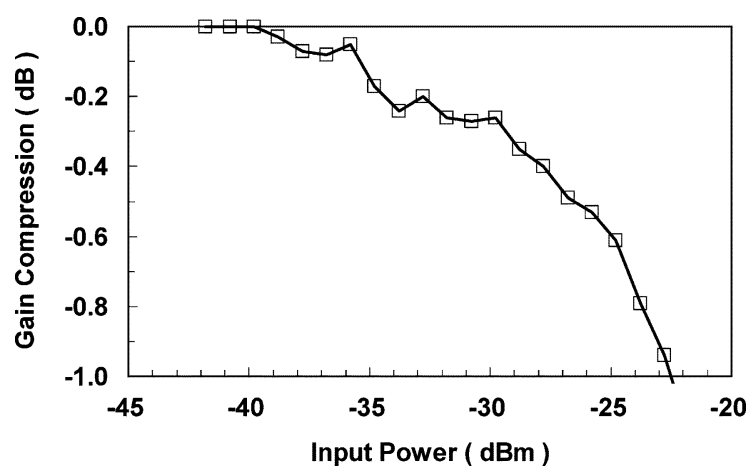

Fig. 17. The gain compression versus input power for measuring input 1-dB compression point.

TABLE V

RECEIVER FRONT-END PERFORMANCE SUMMARY

\begin{tabular}{lc}
\hline Center Frequency & $5.4 \mathrm{GHz}$ \\
Input Return Loss & $-17.4 /-15.5^{*} \mathrm{~dB}$ \\
Noise Figure & $7.2 / 5.2^{*} \mathrm{~dB}$ \\
Input IP & $-12.5 \mathrm{dBm}$ \\
Voltage Gain (high) & $25.2 / 26.2^{*} \mathrm{~dB}$ \\
Voltage Gain (low) & $1.4 \mathrm{~dB}$ \\
LO-rejection & $54.9 / 68.9^{*} \mathrm{~dB}$ \\
LO Leakage & $-55.9 /-83.2^{*} \mathrm{dBm}$ \\
2XLO Leakage & $-88.7 /-120.1^{*} \mathrm{dBm}$ \\
Power Dissipation & $45.5 \mathrm{~mW}$ \\
Supply Voltage & $1.0 \mathrm{~V}$ \\
Technology & $0.18-\mathrm{um} \mathrm{CMOS}$
\end{tabular}

*: after ICP post process

$20 \mathrm{MHz}$ baseband is $7.0 \mathrm{~dB}$ and $5.2 \mathrm{~dB}$ before and after the CMOS-compatible ICP post process. As expected, the circuit is LO self-mixing free due to the new-proposed SHM topology. The achieved input-referred DC-offset due to LO self-mixing is below $-110.7 \mathrm{dBm}$.

\section{ACKNOWLEDGMENT}

Chip fabrication from UMC under campus program and technical help from CIC and NDL are appreciated.

\section{REFERENCES}

[1] M. V. Schneider and W. W. Snell, "Harmonically pumped stripline down-converter," IEEE Trans. Microw. Theory Tech., vol. MTT-23, pp. 271-275, Mar. 1975.

[2] M. Cohn, J. E. Degenford, and B. A. Newman, "Harmonic mixing with an anti-parallel diode pair," IEEE Trans. Microw. Theory Tech., vol. MTT-23, pp. 667-673, Aug. 1975.
[3] T. Yamaji, H. Tanimoto, and H. Kokatsu, "A I/Q active balanced harmonic mixer with IM2 cancellers and a $45^{\circ}$ Phase shifter," IEEE $J$. Solid-State Circuits, vol. 33, no. 12, pp. 2240-2246, Dec. 1998.

[4] Z. Zhang, Z. Chen, and J. Lau, "A 900 MHz CMOS balanced harmonic mixer for direct conversion receivers," in Proc. IEEE RAWCON, Sep. 2000, pp. 219-222.

[5] L.-W. Sheng, J. C. Jensen, and L. E. Larson, "A wide-bandwidth $\mathrm{Si} / \mathrm{SiGe}$ HBT direct conversion subharmonic mixer/downconverter," IEEE J. Solid-State Circuits, vol. 35, no. 9, pp. 1329-1337, Sep. 2000.

[6] R. Svitek and S. Raman, "5-6 GHz SiGe active I/Q subharmonic mixers with power supply noise effect characterization," IEEE Microw. Wireless Compon. Lett., vol. 14, no. 7, pp. 319-321, Jul. 2004.

[7] M. Goldfarb, E. Balboni, and J. Cavey, "Even harmonic double-balanced active mixer for use in direct conversion receivers," IEEE J. Solid-State Circuits, vol. 38, no. 10, pp. 1762-1766, Oct. 2003.

[8] K. J. Koh, M. Y. Park, C. S. Kim, and H. K. Yu, "Subharmonically Pumped CMOS frequency conversion (up and down) circuits for 2-GHz WCDMA direct-conversion transceiver," IEEE J. Solid-State Circuits, vol. 39, no. 6, pp. 871-884, Jun. 2004.

[9] H.-C. Chen, T. Wang, G.-W. Huang, and S.-S. Lu, "A monolithic 5.9 $\mathrm{GHz}$ CMOS I/Q direct-down converter utilizing a quadrature coupler and transformer-coupled subharmonic mixers," IEEE Microw. Wireless Compon. Lett., vol. 16, no. 4, pp. 197-199, Apr. 2006.

[10] E. Abou-Allam, J. J. Nisbet, and M. C. Maliepaard, "Low-voltage 1.9-GHz front-endreceiver in 0.5- $\mu \mathrm{m}$ CMOS technology," IEEE J. Solid-State Circuits, vol. 36, no. 10, pp. 1434-1443, Oct. 2001.

[11] T. Wang, H.-C. Chen, H.-W. Chiu, Y.-S. Lin, G.-W. Huang, and S.-S. $\mathrm{Lu}$, "Micromachined CMOS LNA and VCO by CMOS-compatible ICP deep trench technology," IEEE Trans. Microw. Theory Tech., vol. 54, no. 2, pp. 580-588, Feb. 2006

[12] G. F. Avitabile, A. Cidronali, C. Salvador, and M. Speciale, "A compact MMIC $90^{\circ}$ coupler for ISM applications," in IEEE MTT-S Dig., Jun. 1997, vol. 1, pp. 281-284.

[13] R. Ahola, A. Aktas, J. Wilson, K. R. Rao, F. Jonsson, I. Hyyryläinen, A. Brolin, T. Hakala, A. Friman, T. Mäkiniemi, J. Hanze, M. Sandén, D. Wallner, Y. Guo, T. Lagerstam, L. Noguer, T. Knuuttila, P. Olofsson, and M. Ismail, "A single-chip CMOS transceiver for $802.11 \mathrm{a} / \mathrm{b} / \mathrm{g}$ wireless LANs," IEEE J. Solid-State Circuits, vol. 39, no. 12, pp. 2250-2258, Dec. 2004.

[14] I. Vassiliou, K. Vavelidis, T. Georgantas, S. Plevridis, N. Haralabidis, G. Kamoulakos, C. Kapnistis, S. Kavadias, Y. Kokolakis, P. Merakos, J. C. Rudell, A. Yamanaka, S. Bouras, and I. Bouras, "A single-chip digitally calibrated $5.15-5.825-\mathrm{GHz} 0.18-\mu \mathrm{m}$ CMOS transceiver for 802.11 a Wireless LAN," IEEE J. Solid-State Circuits, vol. 38, no. 12, pp. 2221-2231, Dec. 2003.

[15] Y.-H. Hsieh, W.-Y. Hu, S.-M. Lin, C.-L. Chen, W.-K. Li, S.-J. Chen, and D.-J. Chen, "An auto-I/Q calibrated CMOS transceiver for 802. $11 \mathrm{~g}$," in IEEE ISSCC Dig. Tech. Papers, 2005, pp. 92-93.

[16] O. Charlon, M. Locher, H. Visser, D. Duperray, J. Chen, M. Judson, A. L. Landesman, C. Hritz, U. Kohlschuetter, Y. Zhang, C. Ramesh, A. Daanen, M. Gao, S. Haas, V. Maheshwari, A. Bury, G. Nitsche, A. Wrzyszcz, W. Redman-White, H. Bonakdar, R. E. Waffaoui, and M. Bracey, "A low-power high-performance SiGe BiCMOS 802.11a/b/g transceiver IC for cellular and Bluetooth co-existence applications," IEEE J. Solid-State Circuits, vol. 41, no. 7, pp. 1503-1512, Jul. 2006.

[17] A. L. L. Pun, T. Yeung, J. Lau, F. J. R. Clement, and D. K. Su, "Substrate noise coupling through planar spiral inductor," IEEE J. SolidState Circuits, vol. 33, no. 6, pp. 877-884, Jun. 1998.

[18] H. Darabi and A. A. Abidi, "Noise in RF-CMOS mixers: A simple physical model," IEEE J. Solid-State Circuits, vol. 35, no. 1, pp. 15-25, Jan. 2000.

[19] B. Razavi, "A 5.2-GHz CMOS receiver with 62-dB image rejection," IEEE J. Solid-State Circuits, vol. 36, no. 5, pp. 810-815, May 2000.

[20] T. Lee, The Design of CMOS RF IC, 2nd ed. Cambridge, U.K.: Cambridge Univ. Press, ch. 11, pp. 344-347.

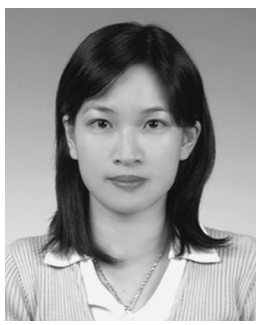

Hsiao-Chin Chen received the B.S. and M.S. degrees in electrical engineering from National Taiwan University, Taipei, Taiwan, R.O.C., in 1998 and 2000, respectively. From 2000 to 2003, she was with Airoha Technology Corp. as an RF IC Design Engineer. Currently, she is working toward the $\mathrm{Ph} . \mathrm{D}$. degree in the Graduate Institute of Electronics Engineering, National Taiwan University.

Her major research interests are RF IC and low-power transmitter design for wireless local area sensor networks. 


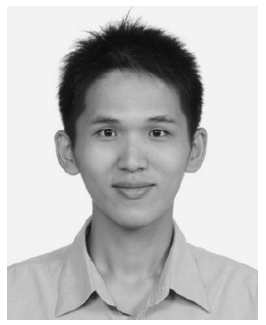

Tao Wang received the B.S. degree from Chang Gung University, Taoyuan, Taiwan, R.O.C., in 2002, and the M.S. degree from National Taiwan University, Taipei, Taiwan, R.O.C., in 2004, both in electronics engineering. He is currently working toward the $\mathrm{Ph} . \mathrm{D}$. degree in electronics engineering at the National Taiwan University.

His research interests are in the areas of radio-frequency integrated circuits and MEMS.

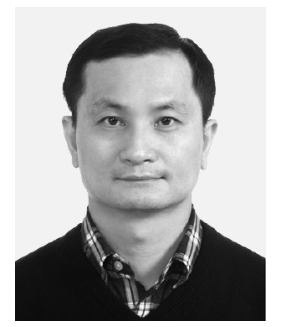

Shey-Shi Lu (S'89-M'91-SM'99) received the B.S. degree from National Taiwan University, Taipei, Taiwan, R.O.C., in 1985, the M.S. degree from Cornell University, Ithaca, NY, in 1988, and the $\mathrm{Ph}$.D. degree from the University of Minnesota, Minneapolis, MN, in 1991, all in electrical engineering.

$\mathrm{He}$ joined the Department of Electrical Engineering, National Taiwan University, in August 1991 as an Associate Professor and was promoted to a full Professor in 1995. His current research interests are in the areas of RFIC/MMICs, micromachined RF components/circuits, and wireless sensor networks. 